\title{
Structural deformation of PVDF nanoweb due to electrospinning behavior affected by solvent ratio
}

https://doi.org/10.1515/epoly-2018-0037

Received February 23, 2018; accepted March 21, 2018; previously published online April 21, 2018

\begin{abstract}
In this study, we investigated the effects of solution characteristics on electrospinning behavior and nanoweb structure. We prepared a polyvinylidene fluoride (PVDF) solution by varying the ratios of $\mathrm{N}, \mathrm{N}$-dimethylformamide (DMF) and acetone in the solvent and fabricated nanowebs by electrospinning. We determined the solution characteristics of the PVDF solution with respect to the acetone ratio in the solvent. The effects of the solution characteristics on the electrospinning behavior were confirmed using a high-speed camera. We found significant differences in the electrospinning behavior. Thus, we investigated the effect of this difference on the morphology and structural properties of the fabricated nanoweb.
\end{abstract}

Keywords: deposition; dynamic behavior; elongation; high-speed camera; thickness.

\section{Introduction}

Polyvinylidene fluoride (PVDF) is an organic material which has excellent chemical resistance, heat resistance, durability, flexibility and biocompatibility $(1,2)$. In addition, PVDF possesses various crystal structures contributed by the conformation of fluorine and hydrogen that are periodically bonded to the polymer chain $(3,4)$. In particular, the $\beta$-phase crystal structure of PVDF is a conformation in which intramolecular polarization is maximized, resulting in excellent electrical characteristics $(5,6)$. In addition, when PVDF is manufactured in the form of nanofibers, it has a large specific surface area. By exploiting the advantages of PVDF, it has been employed in many applications such as in filter materials, various sensors, secondary battery separators and cell scaffolds. In addition, PVDF can be replaced with a conventional

\footnotetext{
*Corresponding author: Han Seong Kim, Department of Organic Material Science and Engineering, Pusan National University, Busan, Republic of Korea, e-mail: hanseongkim@pusan.ac.kr Young Jin Hwang and Sejin Choi: Department of Organic Material Science and Engineering, Pusan National University, Busan, Republic of Korea
}

ceramic piezoelectric device or can be used as a material in wearable devices (7-12).

Among the methods of fiberizing PVDF, electrospinning is the most popular method because it is easy to obtain a $\beta$-phase crystal structure using this method. Electrospinning is a technique for producing nanofibers using the electrostatic force between a polymer solution droplet and a collector (13). During the process, the $\beta$-phase crystal structure of the PVDF is induced by the stretching action of the polymer and the electric field (14). As the process is simple, it is easy to install, thus facilitating mass production with low cost $(15,16)$.

Although the principle of electrospinning is simple, the spinning mechanism is complicated; studies on the stability analysis have been conducted (17). Electrospinning is affected by process parameters such as the nozzle diameter, applied voltage and tip-to-collector distance (TCD) (18-20). Moreover, it is influenced by solution properties such as concentration, viscosity, surface tension, vapor pressure and dielectric constant (21-23). Therefore, to establish stable spinning conditions and to control the fiber structure, the effects of these conditions must be understood.

In this study, the effects of solvent ratio on the electrospinning behavior and the characteristics of the collected nanoweb were compared and analyzed by investigating the solution characteristics such as viscosity, surface tension and volatility with respect to the ratio of acetone in the solvent. During the electrospinning process, the changes in the droplet and jet behaviors with respect to the solution characteristics were observed using a chargecoupled device (CCD) camera, and the effects of these changes on the morphology and three-dimensional structure of the nanoweb were analyzed.

\section{Experimental procedure}

\subsection{Materials}

$N, N$-dimethylformamide (DMF, Junsei Chemical Co. Ltd., Tokyo, Japan) and acetone (Duksan pure chemicals Co. Ltd., Ansan, Republic of Korea) were mixed with various DMF:acetone weight ratios, such as 10:0, 8:2, 6:4, 4:6 and 2:8, as a solvent. PVDF powder (Mw: 534,000, 
Sigma-Aldrich Inc., Saint Louis, MO, USA) was dissolved in the DMF:acetone solvent mixtures with a concentration of $15 \mathrm{wt} \%$, and the mixture was magnetically stirred at $55^{\circ} \mathrm{C}$ for $6 \mathrm{~h}$. The prepared solutions were denoted as $\mathrm{A} 0$, $\mathrm{A} 2, \mathrm{~A} 4, \mathrm{~A} 6$ and $\mathrm{A} 8$ with respect to the acetone content in the mixed solvents.

\subsection{Electrospinning setup}

The PVDF solution was fed into a metal nozzle with an inner diameter of $0.495 \mathrm{~mm}(21 \mathrm{G})$ at a pressure of $20 \mathrm{kPa}$ by compressed air controlled by the regulator to maintain a constant feed rate. In this condition, the flow rates were measured at 4.14(A0), 5.81(A2), 9.47(A4), 12.86(A6), $14.21 \mathrm{ml} / \mathrm{h}$ (A8), respectively. A high voltage of $13 \mathrm{kV}$, generated by a high-voltage power supply (ESN-HV30, NanoNC Co. Ltd., Seoul, Republic of Korea), was applied to the nozzle, forming the same electric field between the nozzle and the grounded metal collector. The TCD was fixed at $15 \mathrm{~cm}$. The resulting deposition, i.e. nanoweb, was collected during the electrospinning process for $10 \mathrm{~min}$ at the room temperature of $20^{\circ} \mathrm{C}$ and the relative humidity of $50 \%$, and subsequently dried for $12 \mathrm{~h}$.

\subsection{Characterization}

The viscosity, conductivity and surface tension of the PVDF solutions were measured using a viscometer (DV-II+Pro, AMETEK Brookfield Inc., MA, USA), a conductivity meter (HI 8633, HANNA Instruments Inc., Rhode Island, USA), and a surface tension meter (SEO-DST30M, SEO Ltd., Suwon, Republic of Korea), respectively, to determine the solution properties. The images of the drop hanging from the vertex of the nozzle tip (Figure 1A); and the jet, which is the entire material between the bottom of the drop and the collector (Figure 1B), were captured using a CCD camera (SCC-B2315, Samsung Electronics Co., Suwon, Republic of Korea) for $600 \mathrm{~s}$ with an interval of $1 \mathrm{~s}$ to investigate the dynamic behavior of electrospinning. The original images of the drop and jet were converted to black-and-white images by adjusting the critical value of the image intensity. The changes in the drop and jet areas with time were determined using a self-programmed image analysis tool. In addition, a high-speed camera (Phantom Miro eX2, Vision Research Inc., NJ, USA) was used to capture the jet behavior in a more detailed manner.

The grayscale images of the deposited nanoweb, captured using a scanner (PIXMA E510, Canon Inc., Tokyo, Japan), were converted into corresponding contour images to analyze the distribution of the deposition. The

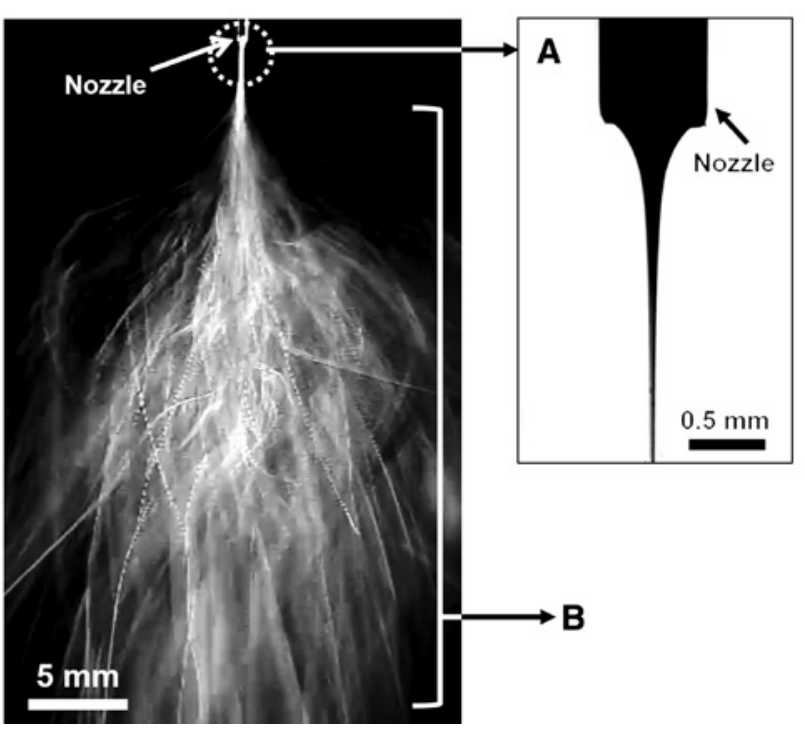

Figure 1: Images of (A) drop and (B) jet area, captured using the CCD camera during the electrospinning process.

morphologies of the nanoweb were observed using a fieldemission scanning electron microscope (SUPRA25, Carl Zeiss Co. Ltd., Oberkochen, Germany), and the diameters of the nanofibers were calculated using a software (i-Solution Lite, IMT i-Solution Inc., Vancouver, Canada). The compression test conducted on the nanoweb was evaluated using a universal testing machine (Texture Analyzer, Yeonjin Corp., Seoul, Republic of Korea) by applying a compression load at a rate of $0.1 \mathrm{~mm} / \mathrm{s}$.

\section{Results and discussion}

The electrospinning behavior is significantly affected by the applied electrical force and solution properties including surface tension, viscosity and conductivity. Therefore, the solution properties are determined, as listed in Table 1. Both the surface tension and the shear viscosity gradually decrease from 39.7 dyne/cm and $2549.5 \mathrm{cP}$ to 24.8 dyne/ $\mathrm{cm}$ and $959.8 \mathrm{cP}$, respectively, with the increase in the acetone ratio in the solvent, because acetone weakens

Table 1: Characteristics of PVDF solutions used in this study.

\begin{tabular}{lrrr}
\hline & Surface tension (dyne/cm) & Viscosity (cP) & Conductivity $(\boldsymbol{\mu s})$ \\
\hline A0 & 39.7 & 2549.5 & 13 \\
A2 & 34.9 & 1889.6 & 16.3 \\
A4 & 29.7 & 1499.7 & 17.7 \\
A6 & 27.4 & 1154.8 & 20.7 \\
A8 & 24.8 & 959.8 & 24.4 \\
\hline
\end{tabular}


the molecular interaction between the components of the PVDF solution, as it is a non-solvent additive (24). The conductivity gradually increases up to $24.4 \mu \mathrm{s} / \mathrm{cm}$ for A8 solution with the increase in the amount of acetone, implying that A8 can be electrically charged very easily.

The instability of the drop hanging from the vertex of the nozzle tip was investigated, as it is the first step in the electrospinning process. The change in the drop area, which represents the amount of droplet, is decided by the balance between the rate of solution flowing out from the nozzle and the rate of jet ejecting from the drop. The rate of solution flowing out is affected by the shear viscosity of the solution, while the rate of jet ejection is affected by the surface tension and the charge repulsion force, induced
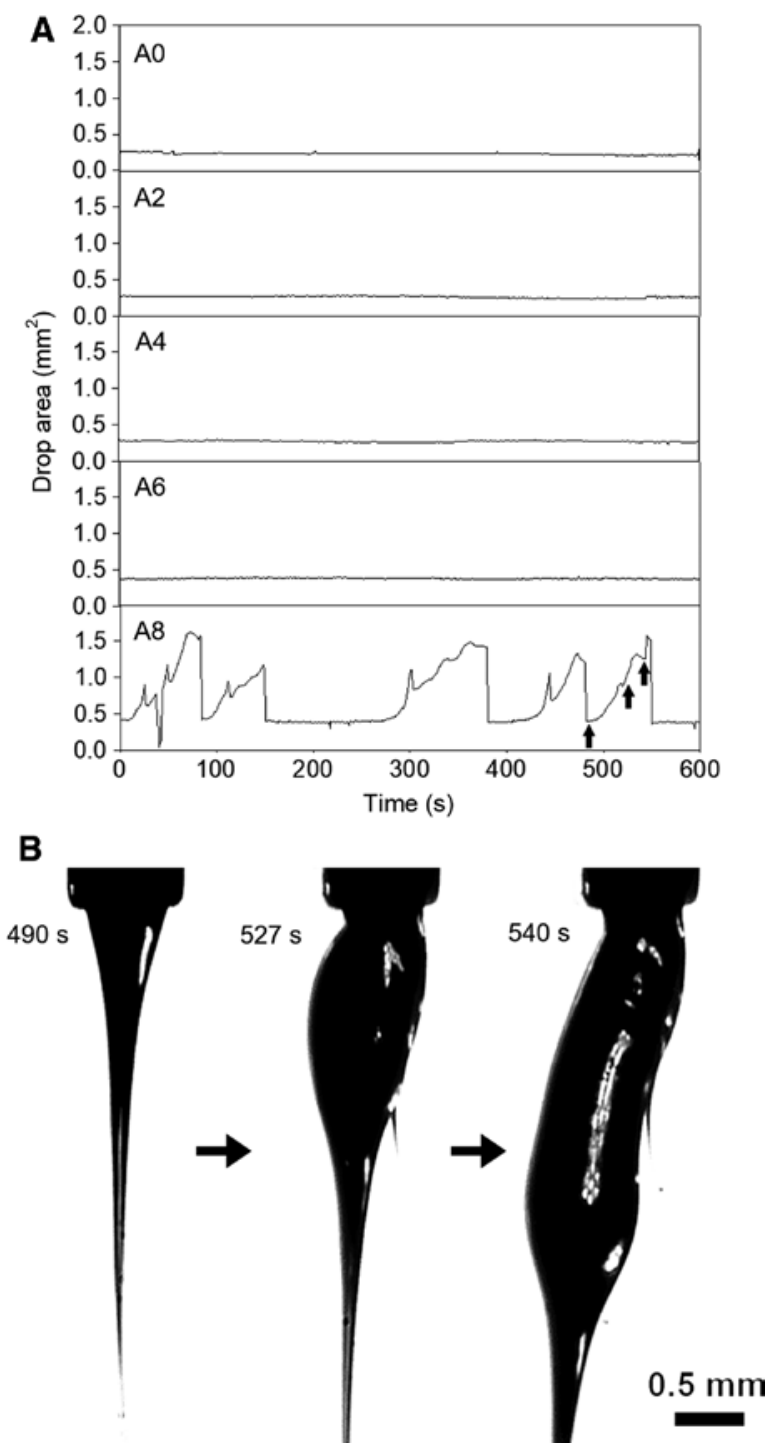

Figure 2: (A) Change in the drop area during the electrospinning process for $600 \mathrm{~s}$, and (B) corresponding images of the drop change for $A 8$, indicated by black arrows in (A). by an applied voltage of $13 \mathrm{kV}$. As shown in Figure 2A, even though the shear viscosity decreases with the increase in the acetone ratio, the drop areas corresponding to $\mathrm{A} 0, \mathrm{~A} 2$, A4 and A6 solutions remain constant with respect to time at the same applied voltage, because of the decrease in the surface tension. However, for the A8 solution, the drop area changes unstably with time. When the acetone proportion in the solvent is extremely high, the PVDF solution rapidly solidifies because of the high evaporating rate of acetone, which has a high vapor pressure (25).

The electrospun jet area represents the amount of solution ejected from the drop. Moreover, the jet shape affects the formation of the resultant deposition. The change in the jet area is one of the important parameters

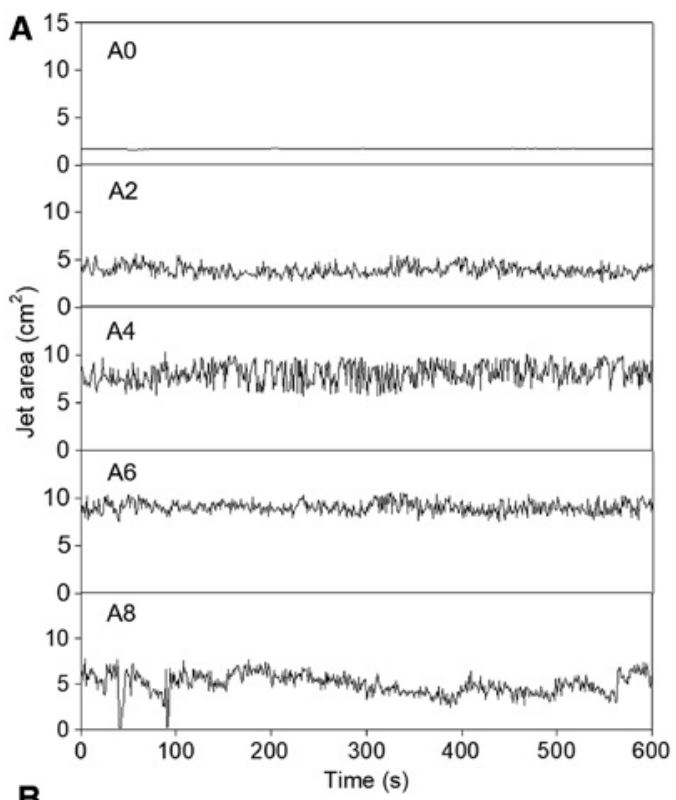

B
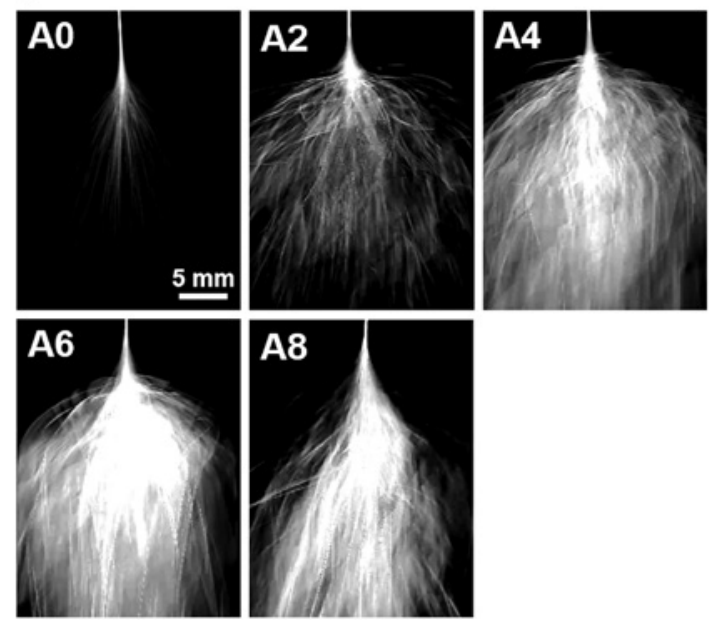

Figure 3: (A) Change in the jet area during the electrospinning process for $600 \mathrm{~s}$, and (B) corresponding jet images captured using the CCD camera. 
to be investigated. The average jet area increases with the increase in the acetone ratio up to A6, as shown in Figure 3A. The increased amount of jet was because of the higher rate of jet ejection due to the gradually decreasing surface tension and the increasing chargeability of the solution at the same applied voltage with the increase in the acetone ratio, as mentioned previously. However, the jet amount for A8 decreases slightly, and the reason can be seen in Figure 3B. It was confirmed that the density of the jet for the same TCD increases, from A0 to A6; however, the jet for $\mathrm{A} 8$ formed in a smaller region with a narrower spinning angle. Moreover, the unstable change in the jet area for A8 was affected by the unstable drop change, as shown in Figure 2.

To observe the dynamic behavior, the jet shapes were captured using a high-speed camera. In Figure 4, the jet behavior differs markedly with respect to the acetone ratio in the solution. The jets of $\mathrm{A} 0$ and $\mathrm{A} 2$ solutions were broken while stretching, and a fibrous image was rarely observed, as shown in Figure 4A and B. However, with the increase in the acetone content, the breakage phenomenon is reduced, and a clear fibrous image appears. The friction between the PVDF molecules was not high enough to overcome the drawing force, resulting in jet breaking. When acetone is added, the driving jet is solidified rapidly before it exceeds the elongation limit because of the increased volatility of the solvent. In addition, as the elongation rate decreases because of the rapid solidification, the bending radius of the whipping jet becomes smaller, as shown in Figure 4C-E.

Figure 5 shows the contour analysis of the deposition of the spun solutions, obtained using threshold processing. The gray scale light intensities of the image were filtered in 10 steps from 60 to 240 for evaluating the distribution of the deposition (26). In the graph shown in Figure $5 \mathrm{~A}$, the area filtered at 60 (minimum intensity of the fiber) was found to be the widest for $\mathrm{A} 2$ and the narrowest for the acetone-added solution. This can also be seen in the image shown in Figure 5B. This is because of the reduction in the bending radius of the jet due to the elongation difference, as shown in the high-speed camera image analysis (Figure 4). The area of A2 is 0 when the intensity
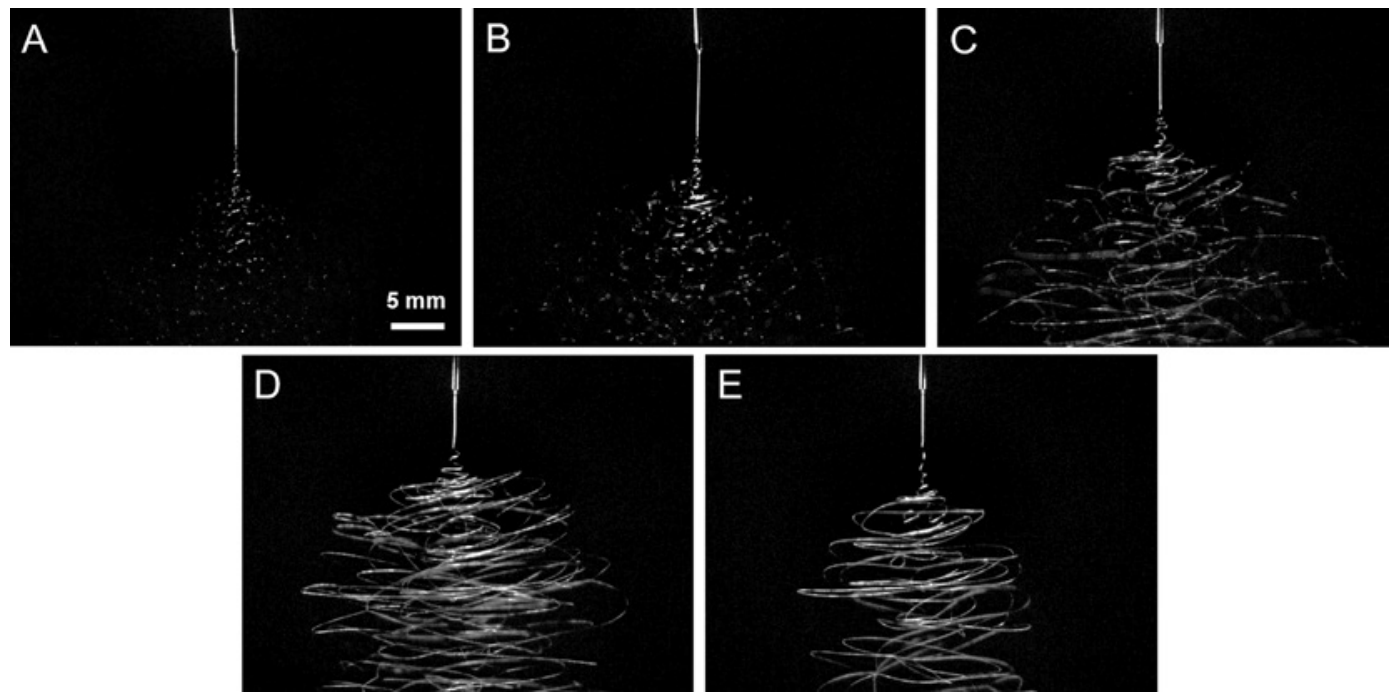

Figure 4: The jet images of (A) A0, (B) A2, (C) A4, (D) A6 and (E) A8, captured using the high-speed camera with an exposure time of $20 \mu \mathrm{s}$.
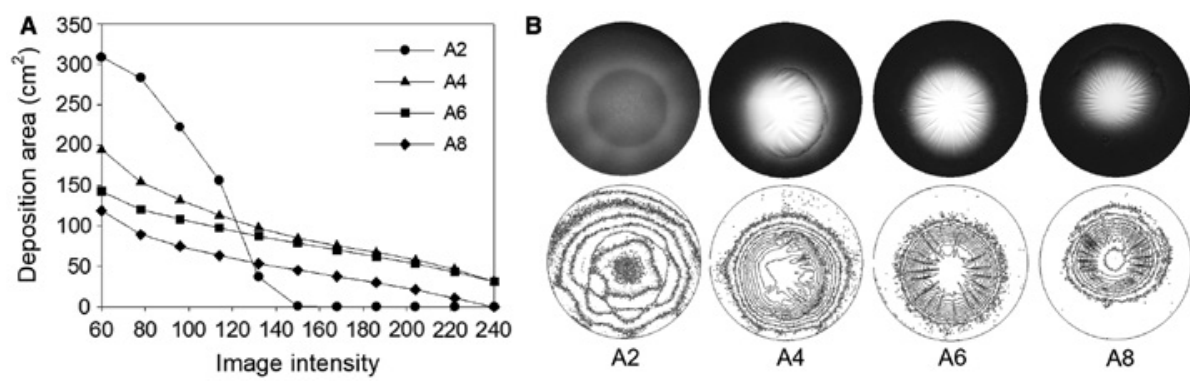

Figure 5: (A) Contour analysis of web deposition through threshold processing and (B) corresponding web images. 
is above 168. The intensity represents the fiber density, and the fact that the area reached zero at a lower intensity compared to that of the other samples shows that the web was not collected more densely than the other samples.

Figure 6 shows the morphology changes with respect to the acetone content. The A0 solution was collected in the form of a film shape and not as a fiber, and the A2 solution was collected in a mixed deposition of beads and fibers, resulting from the jet breaking. However, beads did not appear in A4, which had a higher acetone ratio. This is because of the difference in the jet behavior depending on the solution characteristics (Figure 4), and it was confirmed that the increase in the proportion of acetone improves the fiber formation.
Figure 7 shows the fiber diameter distribution of the electrospun nanowebs. The average diameters of the electrospun nanowebs of A2, A4, A6 and A8 are $0.73 \mu \mathrm{m}$, $1.25 \mu \mathrm{m}, 1.82 \mu \mathrm{m}$ and $2.03 \mu \mathrm{m}$, respectively. It was found that the increased ratio of acetone led to an increase in the fiber diameter, because the volatility of acetone restricts the jet elongation, thus increasing the diameter.

Figure 8 shows the cross-sectional Field emission scanning electron microscopy (FE-SEM) image for the thickness analysis of the fabricated nanowebs. The thicknesses of the nanowebs of A2, A4, A6 and A8 were found to be $12.2 \mu \mathrm{m}, 44.4 \mu \mathrm{m}, 192.2 \mu \mathrm{m}$ and $397.8 \mu \mathrm{m}$, respectively, despite the same collecting time. The increase in the thickness is due to the increase in the amount of jet and
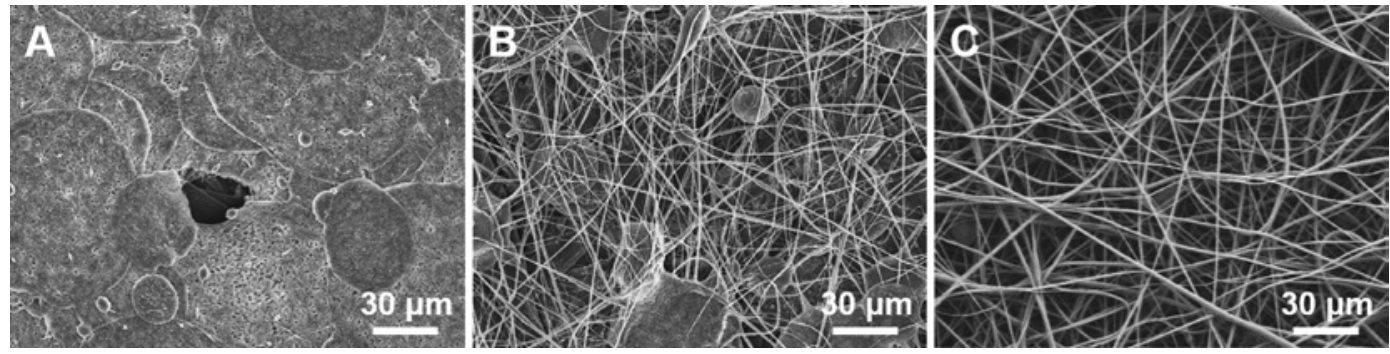

Figure 6: FE-SEM images of nanoweb obtained by electrospun: A) A0, B) A2, and C) A4.
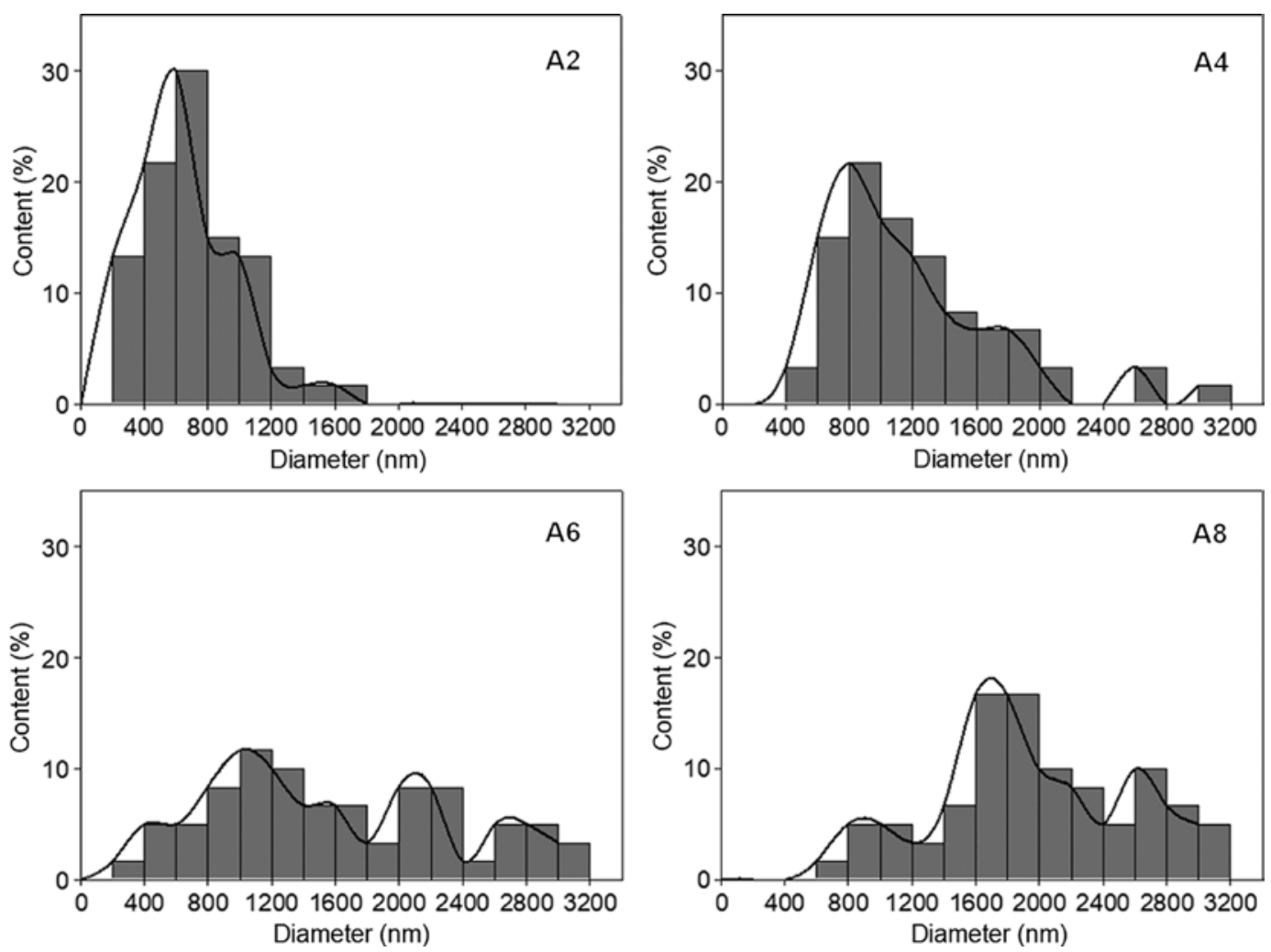

Figure 7: Fiber diameter distribution of electrospun nanowebs. 


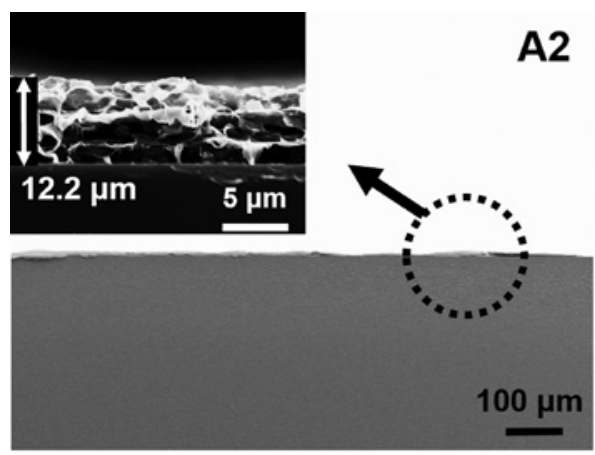

A6
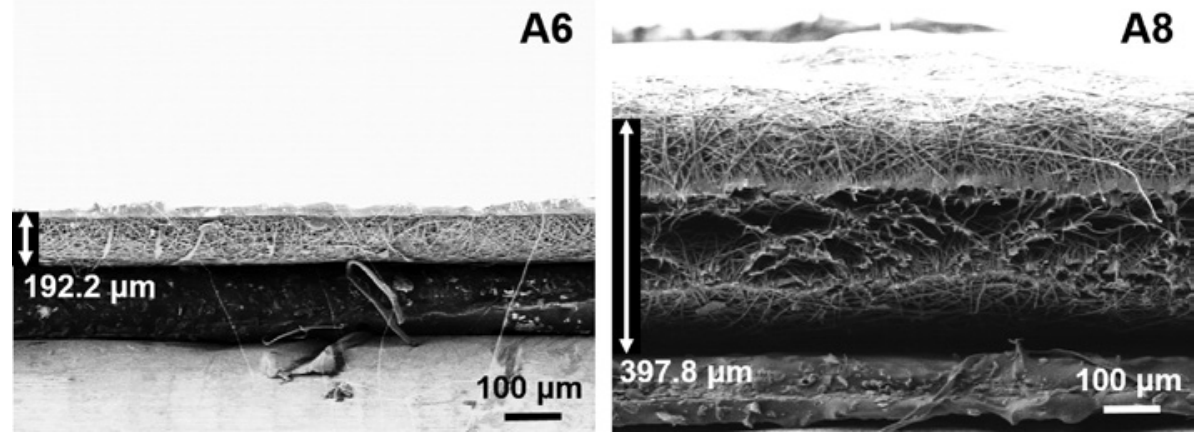

Figure 8: Cross sectional FE-SEM image of electrospun nanoweb and thickness analysis.

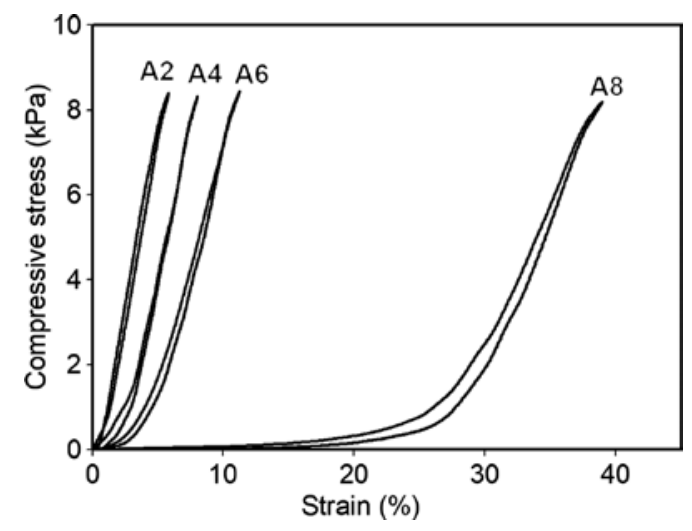

Figure 9: Stress-strain curve of nanowebs under compression.

the average diameter of the fibers with the increase in the ratio of acetone. Also, the thickness of web is affected by the deposition area, and a relatively large amount of fibers is stacked in the small area (Figure 5). Moreover, with the increase in the ratio of acetone, the fibers become more solidified when they are stacked on the collector because of the high volatility, thus the stiff fibers could make the web thicker.

The higher number and greater thickness (of the fibers) of the resultant nanoweb were responsible for the formation of a more porous structure, exhibiting a different response in terms of the compressive property, as shown in Figure 9. The strain was greater when the ratio of acetone was higher at the same compressive stress.

\section{Conclusions}

In this study, we examined the effect of varying the solvent on the electrospinning behavior and nanoweb structure for the fabrication of PVDF nanowebs by electrospinning. The solution characteristics differed depending on the ratio of acetone in the solvent. Moreover, it was found that the solution characteristics are important factors affecting the drop and jet behaviors during electrospinning, causing significant changes in the morphology, fiber diameter, and three-dimensional structural properties of the nanowebs. In summary, this study demonstrates that it is possible to establish stable electrospinning conditions and to control the structure of the nanoweb by selectively controlling the properties of the solvent.

Acknowledgments: This research was supported by a grant from the Individual Basic Science \& Engineering Research Program (NRF-2015R1D1A1A01057302) funded by Ministry of Education, Republic of Korea.

\section{References}

1. Andrew JS, Clarke DR. Effect of electrospinning on the ferroelectric phase content of polyvinylidene difluoride fibers. Langmuir 2008;24(3):670-2. 
2. Rocha JG, Gonclaves LM, Rocha PF, Lanceros-Mendez S. Energy harvesting from piezoelectric materials fully integrated in footwear. IEEE T Ind Electron. 2010;57(3):813-9.

3. Mohammadi B, Yousefi AA, Bellah SM. Effect of tensile strain rate and elongation on crystalline structure and piezoelectric properties of PVDF thin films. Polym Test 2007;26(1):42-50.

4. Lovinger AJ. Poly(vinylidene fluoride). Dordrecht: Springer; 1982. pp. 195-273.

5. Salimi A, Yousefi AA. FTIR studies of $\beta$-phase crystal formation in stretched PVDF films. Polym Test 2003;22(6):699-704.

6. Salamone JC. Polymeric materials encyclopedia. New York: CRC Press; 1996. pp. 7115-7122.

7. Li H, Chen YM, Ma XT, Shi JL, Zhu BK, Zhu LP. Gel polymer electrolytes based on active PVDF separator for lithium ion battery. I: Preparation and property of PVDF/poly(dimethylsiloxane) blending membrane. J Membrane Sci. 2011;379(1):397-402.

8. Ribeiro C, Sencadas V, Correia DM, Lanceros-Méndez S. Piezoelectric polymers as biomaterials for tissue engineering applications. Colloid Surface B. 2015;136(1):46-55.

9. Ahn Y, Lim JY, Hong SM, Lee J, Ha J, Choi HJ, Seo Y. Enhanced piezoelectric properties of electrospun poly(vinylidene fluoride)/multiwalled carbon nanotube composites due to high $\beta$-phase formation in poly(vinylidene fluoride). J Phys Chem C. 2013;117(22):11791-9.

10. Lang CH, Fang J, Shao H, Wang HX, Yan GL, Ding X, Lin T. High-output acoustoelectric power generators from poly(vinylidenefluoride-co-trifluoroethylene) electrospun nanononwovens. Nano Energy 2017;35(1):146-53.

11. Dorneanu PP, Cojocaru C, Olaru N, Samoila P, Airinei A, Sacarescu L. Electrospun PVDF fibers and a novel PVDF/CoFe204 fibrous composite as nanostructured sorbent materials for oil spill cleanup. App Surf Sci. 2017;424(1):389-96.

12. Huang T, Wang C, Yu H, Wang HZ, Zhang QH, Zhu MF. Human walking-driven wearable all-fiber triboelectric nanogenerator containing electrospun polyvinylidene fluoride piezoelectric nanofibers. Nano Energy 2015;14(1):226-35.
13. Ding B. Electrospinning, fibers and textiles: a new driving force for global development. e-polymers 2017;17(3):209-10.

14. Baji A, Mai YW, Li Q, Liu Y. Electrospinning induced ferroelectricity in poly(vinylidene fluoride) fibers. Nanoscale 2011;3(8):3068-71.

15. Formhals A. Process and apparatus for preparing artificial threads. U.S. Patent 1-975-504; 1934.

16. Li D, Xia YN. Electrospinning of nanofibers: reinventing the wheel? Adv Mater. 2004;16(14):1151-70.

17. Ye NK, Kim HS. Fundamental studies on the electrospinning instability for controlling the electrospinning process. Text Sci Eng. 2012;49(4):250-4.

18. Renker DH, Chun I. Nanometre diameter fibres of polymer, produced by electrospinning. Nanotechnology 1996;7(3):216-23.

19. Liu J, Kumar S. Microscopic polymer cups by electrospinning. Polymer 2005;46(10):3211-4.

20. Fong H, Chun I, Renker DH. Beaded nanofibers formed during electrospinning. Polymer 1999;40(16):4585-92.

21. Choi JW, Lee KH, Hwang SH, Kim JY, Lee SW, Huh WS. Study of polycarbonate/MWNT electrospun nanofiber and its multi-filament application. J Korean Ind Eng Chem. 2008;19(4):413-20.

22. Lee KH, Kim HY, Bang HJ, Jung YH, Lee SG. The change of bead morphology formed on electrospun polystyrene fibers. Polymer 2003;44(14):4029-34.

23. Li D, Xia YN. Fabrication of titania nanofibers by electrospinning. Nano Lett. 2003;3(4):555-60.

24. Fontananova E, Jansen JC, Cristiano A, Curcio E, Drioli E. Effect of additives in the casting solution on the formation of PVDF membranes. Desalination 2006;192(1):190-7.

25. Illangakoon UE, Mahalingam S, Matharu RK, Edirisinghe M. Evolution of surface nanopores in pressurised gyrospun polymeric microfibers. Polymers 2007;9(10):1-11.

26. Kong CS, Yoo WS, Lee KY, Kim HS. Nanofiber deposition by electroblowing of PVA(polyvinyl alcohol). J Mater Sci. 2009;44(4):1107-12. 